\title{
Statistical validation of a solar wind propagation model from 1 to $10 \mathrm{AU}$
}

\author{
Bertalan Zieger, ${ }^{1,2}$ and Kenneth C. Hansen ${ }^{1}$ \\ Received 18 January 2008; revised 22 April 2008; accepted 9 May 2008; published 12 August 2008.
}

[1] A one-dimensional (1-D) numerical magnetohydrodynamic (MHD) code is applied to propagate the solar wind from $1 \mathrm{AU}$ through $10 \mathrm{AU}$, i.e., beyond the heliocentric distance of Saturn's orbit, in a non-rotating frame of reference. The time-varying boundary conditions at $1 \mathrm{AU}$ are obtained from hourly solar wind data observed near the Earth. Although similar MHD simulations have been carried out and used by several authors, very little work has been done to validate the statistical accuracy of such solar wind predictions. In this paper, we present an extensive analysis of the prediction efficiency, using 12 selected years of solar wind data from the major heliospheric missions Pioneer, Voyager, and Ulysses. We map the numerical solution to each spacecraft in space and time, and validate the simulation, comparing the propagated solar wind parameters with in-situ observations. We do not restrict our statistical analysis to the times of spacecraft alignment, as most of the earlier case studies do. Our superposed epoch analysis suggests that the prediction efficiency is significantly higher during periods with high recurrence index of solar wind speed, typically in the late declining phase of the solar cycle. Among the solar wind variables, the solar wind speed can be predicted to the highest accuracy, with a linear correlation of 0.75 on average close to the time of opposition. We estimate the accuracy of shock arrival times to be as high as $10-15$ hours within $\pm 75 \mathrm{~d}$ from apparent opposition during years with high recurrence index. During solar activity maximum, there is a clear bias for the model to predicted shocks arriving later than observed in the data, suggesting that during these periods, there is an additional acceleration mechanism in the solar wind that is not included in the model.

Citation: Zieger, B., and K. C. Hansen (2008), Statistical validation of a solar wind propagation model from 1 to 10 AU, J. Geophys. Res., 113, A08107, doi:10.1029/2008JA013046.

\section{Introduction}

[2] The first validation studies of a 1-D hydrodynamic (HD) solar wind propagation model were carried out by Hundhausen and Gosling [1976] and Gosling et al. [1976], analyzing the evolution of a corotating stream between 1 and 5 AU on the basis of near-Earth IMP 7 and Pioneer 10 solar wind observations. Subsequent generations of MHD codes have been successfully employed in describing the dynamical evolution of the solar wind during its propagation throughout the heliosphere both qualitatively and quantitatively. Most of the earlier works on solar wind propagation made use of the radial alignment of two or more spacecraft, simulating the evolution of corotating interaction regions (CIRs) or other solar wind structures with 1-D MHD models [Dryer et al., 1978a, 1978b; Smith et al., 1981; Whang and Burlaga, 1985, 1986; Du et al., 2007]. Two-dimensional (2-D) MHD simulations of solar

\footnotetext{
${ }^{1}$ Department of Atmospheric, Oceanic and Space Sciences, University of Michigan, Ann Arbor, Michigan, USA.

${ }^{2}$ Also at Geodetic and Geophysical Research Institute, Hungarian Academy of Sciences, Sopron, Hungary.

Copyright 2008 by the American Geophysical Union. 0148-0227/08/2008JA013046
}

wind propagation were made by Burlaga et al. [1985] and Pizzo et al. [1995] and tested with observations from different spacecraft in radial alignment. 2-D simulations are expected to perform better than 1-D simulations especially in the outer heliosphere, where the stream front interaction is primarily a shear flow, often with strong components in both transverse directions [Pizzo, 1991]. The solar wind propagation studies mentioned above were limited to heliocentric distances of less then 5 AU. Wang et al. [2000] were the first to model the evolution of solar wind structures further out in the heliosphere, from Ulysses ( $1-5 \mathrm{AU})$ to Voyager 2 (33-36 AU), including the effect of pickup ions as momentum and energy source terms in the MHD equations. The authors concluded that the presence of pickup ions slows the solar wind, reduces the amplitude of the speed variations and density spikes, and changes the speed of shock propagation. As confirmed by outer heliospheric simulations, the solar wind pickup of interstellar neutrals is negligible within the ionization cavity $(<6-$ 10 AU), does not change the solar wind structures dramatically within $35 \mathrm{AU}$, but becomes an important factor at heliocentric distances around 50-60 AU [Wang et al., 2001a, 2001b; Richardson et al., 2002]. Solar wind predictions with MHD models have received renewed interest 
recently in connection with the outer planet missions of Galileo and Cassini, and the Hubble Space Telescope campaigns of planetary aurora observations [Hanlon et al., 2004a, 2004b; Prangé et al., 2004; Tao et al., 2005]. Tao et al. [2005] made solar wind dynamic pressure predictions in the foreshock region of Jupiter, validating their 1-D MHD model with 2 years of Ulysses observations. On the basis of mostly qualitative assessment of prediction efficiency and a very limited statistical analysis of shock arrival times (12 selected cases), they found that large pressure pulses are reasonably well predicted if the separation of Earth and Ulysses is less than $50^{\circ}$ in heliographic longitude, and estimated the error of shock arrival times to be about 20 hours.

[3] The aim of the present paper is to validate our 1-D MHD model of solar wind propagation and to quantify the prediction efficiency variations both in space and time within the heliocentric distance of $10 \mathrm{AU}$ on the basis of the largest currently available statistical sample of heliospheric and corresponding near-Earth solar wind data. No such comprehensive statistical studies of solar wind propagation have been previously performed to the best of our knowledge. Unlike most of the earlier case studies, our analysis is not restricted to the periods of spacecraft alignment or opposition. We also address of questions how the prediction efficiency depends on the heliocentric distance and the solar cycle phase, which is not possible in individual case studies. Finally, we give statistically significant error estimates of predicted shock arrival times, which is essential information for the users of solar wind predictions.

[4] The paper is organized as follows. In section 2, we introduce the 1-D numerical MHD-model of solar wind propagation, explain how the time-dependent inner boundary conditions are obtained from near-Earth solar wind observations and how the solution is mapped to moving spacecraft or celestial bodies. In section 3, we validate the model with heliospheric solar wind observations from the Pioneer, Voyager, and Ulysses spacecraft, and present our statistical analysis of prediction efficiency and shock arrival times. We summarize our results in section 4 .

\section{Solar Wind Propagation Model}

[5] The Versatile Advection Code (VAC) is a general software package designed to solve a conservative system of hyperbolic partial differential equations with additional non-hyperbolic source terms, using different numerical schemes [Tóth, 1996]. VAC has been successfully applied for simulating diverse multidimensional steady-state or time-dependent HD or MHD problems in space physics and astrophysics, including the modeling of transonic stellar winds [Keppens and Goedbloed, 1999a, 1999b, 2000]. Keppens and Goedbloed [1999b] have shown that the steady-state numerical solutions of the 1-D isothermal HD and the polytropic MHD wind models exactly match the classical analytical solutions of the isothermal Parker wind [Parker, 1958] and the magnetized Weber-Davis wind [Weber and Davis, 1967], respectively. VAC has been adapted for 1-D simulations of solar wind propagation from 1 to $10 \mathrm{AU}$ as well, and its numerical solar wind predictions were used in several earlier papers [Hanlon et al., 2004a, 2004b; Prangé et al., 2004]. However this is the first comprehensive validation paper of this 1-D solar wind propagation model. As a validation test of the numerical methods implemented in VAC, we ran a 1-D steady-state simulation with the isothermal MHD module and compared the solution with the analytical Parker wind equation solved with the Newton-Raphson method. We confirmed the expected perfect agreement between the two solutions in the heliocentric range from 1 to $10 \mathrm{AU}$ (not shown here).

[6] For the actual solar wind simulations we use the full MHD module of VAC solving the following ideal MHD equations in the conservative form:

$$
\begin{gathered}
\frac{\partial \rho}{\partial t}+\nabla \cdot(\mathbf{v} \rho)=0 \\
\frac{\partial \rho \mathbf{v}}{\partial t}+\nabla \cdot(\mathbf{v} \rho \mathbf{v}-\mathbf{B B})+\nabla p_{t o t}=0 \\
\frac{\partial e}{\partial t}+\nabla \cdot\left(\mathbf{v} e-\mathbf{B B} \cdot \mathbf{v}+\mathbf{v} p_{t o t}\right)=0 \\
\frac{\partial \mathbf{B}}{\partial t}+\nabla \cdot(\mathbf{v B}-\mathbf{B v})=0,
\end{gathered}
$$

where the total pressure $p_{t o t}$ is introduced as

$$
\begin{gathered}
p_{\text {tot }}=p+\mathbf{B}^{2} / 2, \\
p=(\gamma-1)\left(e-\rho \mathbf{v}^{2} / 2-\mathbf{B}^{2} / 2\right) .
\end{gathered}
$$

[7] The conserved variables are the density $\rho$, the momentum density $\rho \mathbf{v}$, the total energy density $e$, and the magnetic field $\mathbf{B}$. The magnetic field is measured in a normalized unit that gives a magnetic permeability of 1 . The value of the adiabatic index $\gamma$ is set to $5 / 3$ corresponding to a monoatomic ideal gas with three degrees of freedom. The source terms coming from the solar gravitational field are neglected in the momentum and energy conservation equations (2) and (3), respectively. The numerical scheme Total Variation Diminishing Monotonic Upstream Scheme for Conservation Laws (TVD-MUSCL) is used to obtain a robust solution.

[8] The $\nabla \cdot \mathbf{B}=0$ constraint in 1-D simulations does not allow the radial magnetic field component $B_{r}$ to change in time, therefore we apply the theoretical $1 / r^{2}$ relation to $B_{r}$ in the simulation domain, using a typical initial value of $5 \mathrm{nT}$ at $1 \mathrm{AU}$. Tests with slightly different initial values show that the simulation results are not affected significantly by the selection of $B_{r}$. The simulation domain is a uniform 1-D grid of 4,000 cells between 1 and $10 \mathrm{AU}$, yielding a spatial resolution of $2.25 \cdot 10^{-3} \mathrm{AU}$ or $3.36 \cdot 10^{5} \mathrm{~km}$. Having tested the grid convergence, we concluded that this resolution is sufficient for our purposes. Spherical symmetry is assumed in the transverse directions, which means that the quantities in the virtual neighboring cells are the same as in the local cell except for the rotation of vector quantities around the origin. The non-vanishing fluxes entering from 


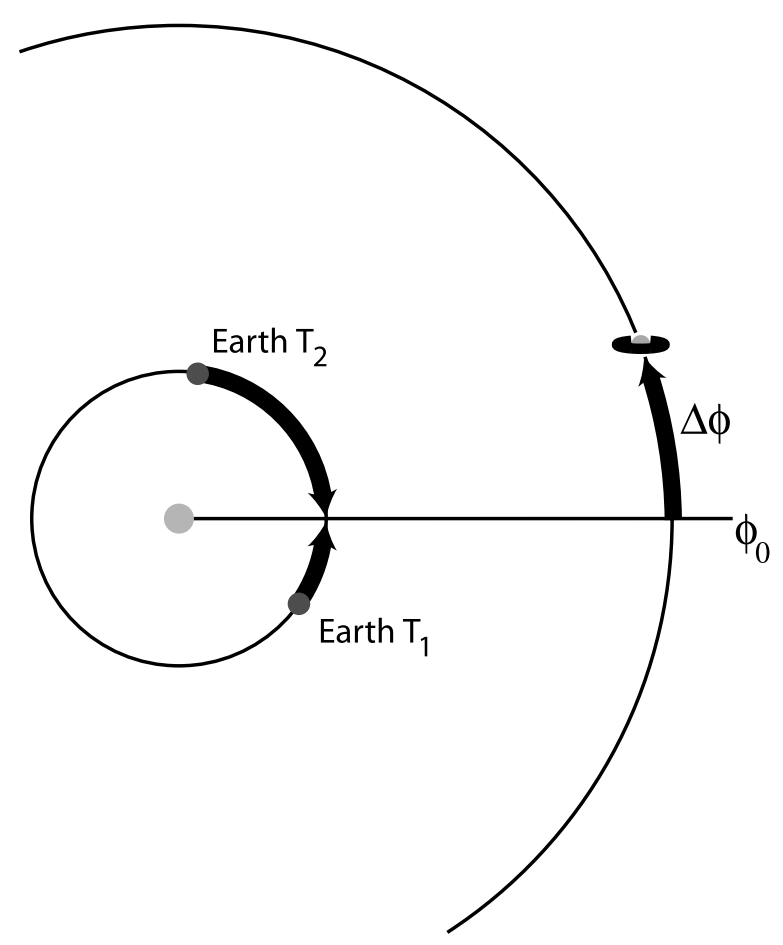

Figure 1. Scheme of mapping the solar wind boundary conditions from Earth to the fixed helioecliptic longitude of the simulation $\left(\Phi_{0}\right)$.

neighboring ghost cells to the local cell are added as geometric source terms to the radial fluxes.

[9] The simulation is performed in an inertial frame fixed to a pre-selected helioecliptic longitude $\Phi_{0}$. The initial conditions for time-dependent runs are obtained from a steady-state solution with average solar wind conditions at the inner boundary to avoid transient numerical effects in the beginning of time-dependent simulations. The timedependent runs are driven by time-dependent boundary conditions at $1 \mathrm{AU}$, specified with the seven observed quantities of density $\rho$, the three vector components of solar wind velocity $\mathbf{v}$ (in RTN coordinates), proton temperature $T$, and two vector components of the magnetic field $B_{T}$ and $B_{N}$. The RTN system is centered at a spacecraft or planet and oriented with respect to the line connecting the Sun and spacecraft or planet. The $\mathrm{R}$ (radial) axis is directed radially away from the Sun through the spacecraft or planet. The T (tangential) axis is the cross product of the Sun's spin vector and the $\mathrm{R}$ axis, and the $\mathrm{N}$ (normal) axis completes the right handed set. As mentioned above, the radial magnetic field component $B_{R}$ has to be kept constant in 1-D numerical simulations because of the $\nabla \cdot \mathbf{B}=0$ constraint. As our code propagates also the transverse components of vector quantities, it is in fact a 1.5-D model rather than a 1-D model in the standard terminology of numerical methods.

[10] Since we do not restrict our simulations to the periods of alignment, or opposition, when the Earth and a given spacecraft are located around the same helioecliptic longitude, we have to estimate the inner boundary conditions of the simulation from near-Earth solar wind observations at helioecliptic longitudes other than $\Phi_{0}$. This is achieved by rotating the boundary conditions observed at Earth forward or backward in time to the longitude $\Phi_{0}$ with the angular velocity of the Sun in the inertial coordinate system (sidereal angular velocity $\Omega_{\text {sid }}$ ), as illustrated in Figure 1. In mathematical terms:

$$
\mathbf{W}\left(t+\left(\Phi_{0}-\Phi_{E}\right) / \Omega_{s i d}, r_{E}, \Phi_{0}\right)=\mathbf{W}\left(t, r_{E}, \Phi_{E}\right),
$$

where $\mathbf{W}$ stands for the array of the 8 MHD variables $(\rho, \mathbf{v}$, $T, \mathbf{B})$, and $r_{\mathrm{E}}$ and $\Phi_{E}$ are the Earth's heliocentric distance and helioecliptic longitude, respectively. A similar procedure can be used to rotate the time dependent 1-D MHD solution $\mathbf{W}\left(t, r, \Phi_{0}\right)$ to any point in space, e.g., to the location of Saturn if we neglect the small displacement of the planet during the time of $\Delta \Phi / \Omega_{\text {sid }}$ (see Figure 1). We can minimize this rotation time by choosing $\Phi_{0}$ at or close to the helioecliptic longitude of the planet.

[11] However, when we are mapping the solution to a fast-moving spacecraft or celestial body (a comet for example), the displacement of the body during the rotation of the solution cannot be neglected. If the spacecraft is located at the ephemeredes $r$ and $\Phi$ at the time $t$, the correctly mapped solution will be

$$
\mathbf{W}\left(t+\left(\Phi^{\prime}-\Phi_{0}\right) / \Omega_{\text {sid }}, r^{\prime}, \Phi^{\prime}\right)=\mathbf{W}\left(t, r^{\prime}, \Phi_{0}\right),
$$

where $r^{\prime}$ and $\Phi^{\prime}$ denote the location of the spacecraft at the time $t+\Delta \Phi^{\prime} / \Omega_{\text {sid }}$, when the forward or backward rotated solution meets the instantaneous helioecliptic longitude of the spacecraft (see illustration in Figure 2). In our model, an iterative procedure is used to find $\Delta \Phi^{\prime}$, interpolating the instantaneous ephemeredes of the spacecraft in each time step.

[12] The mapping procedures described above are based on the assumption that the solar wind boundary conditions on the solar source surface, rotating with the Sun, do not

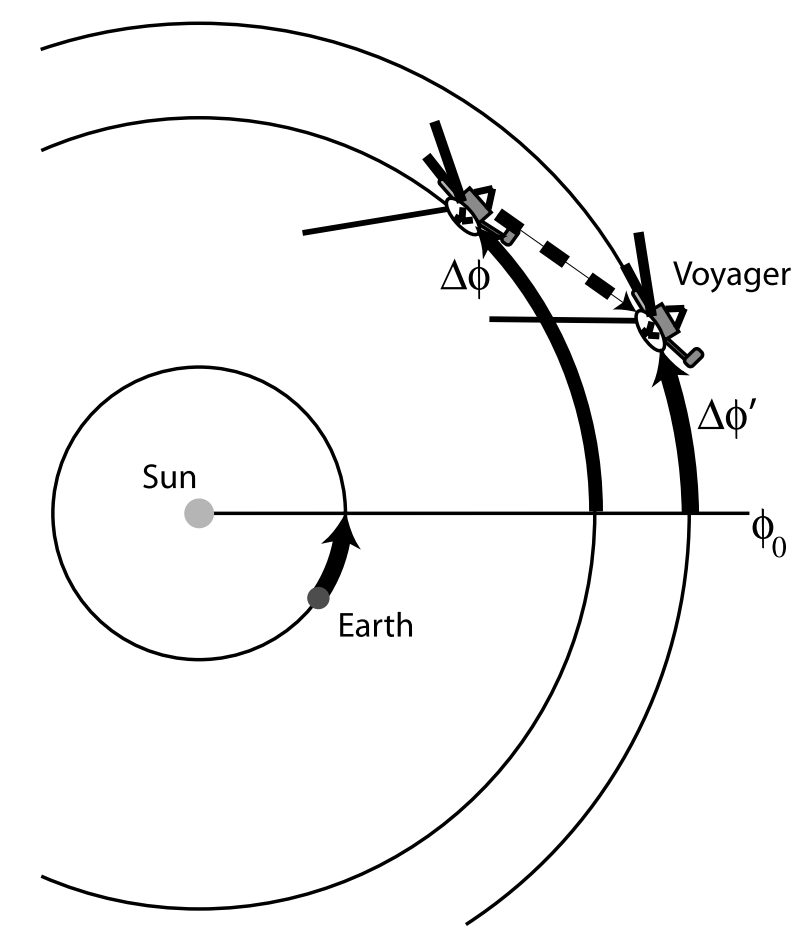

Figure 2. Scheme of mapping the MHD solution to fastmoving spacecraft. 
Table 1. Heliospheric Solar Wind Data Used for Validation Purposes

\begin{tabular}{|c|c|c|c|c|c|c|}
\hline Spacecraft & Period (year) & $\begin{array}{c}\text { Apparent Opposition } \\
\text { (day of year) }\end{array}$ & Range (AU) & $\begin{array}{l}\text { Helioecliptic } \\
\text { Latitude }\left({ }^{\circ}\right)\end{array}$ & $\begin{array}{c}\text { OMNI Data } \\
\text { Coverage }(\%)\end{array}$ & $\begin{array}{c}\text { Recurrence } \\
\text { Index } \\
\end{array}$ \\
\hline Pioneer 10 & $1973-1974$ & 228,1973 & 3.64 to 5.11 & -1.45 to -0.17 & 65 & 0.50 \\
\hline Pioneer 10 & $1974-1975$ & 279,1974 & 5.22 to 7.01 & 0.23 to 2.12 & 63 & 0.72 \\
\hline Pioneer 11 & $1974-1975$ & 264,1974 & 3.67 to 4.46 & -1.74 to 5.71 & 64 & 0.72 \\
\hline Pioneer 11 & $1978-1979$ & 87,1979 & 7.68 to 9.37 & 5.09 to 2.46 & 95 & 0.17 \\
\hline Pioneer 11 & $1979-1980$ & 108,1980 & 9.36 to 9.72 & 2.84 to 8.69 & 83 & 0.20 \\
\hline Voyager 1 & $1978-1979$ & 40,1979 & 3.89 to 5.96 & 0.78 to 1.41 & 94 & 0.24 \\
\hline Voyager 1 & $1979-1980$ & 90,1980 & 6.26 to 9.11 & 1.60 to 2.27 & 81 & 0.15 \\
\hline Voyager 2 & $1978-1979$ & 42,1979 & 3.68 to 5.46 & 2.50 to 0.96 & 94 & 0.24 \\
\hline Voyager 2 & $1979-1980$ & 80,1980 & 5.61 to 7.46 & 1.13 to 2.12 & 83 & 0.15 \\
\hline Voyager 2 & $1980-1981$ & 111,1981 & 7.65 to 9.64 & 2.17 to 2.35 & 84 & 0.05 \\
\hline Ulysses & $1997-1998$ & 72,1998 & 5.26 to 5.34 & 11.79 to -6.11 & 98 & 0.06 \\
\hline Ulysses & $2003-2004$ & 72,2004 & 5.15 to 5.39 & 15.49 to -2.85 & 94 & 0.40 \\
\hline
\end{tabular}

change significantly during the rotation of the boundary conditions at $1 \mathrm{AU}$ to the fixed longitude $\Phi_{0}$ or during the rotation of the solution from $\Phi_{0}$ to the location of the satellite or planet. In other words, we assume that the solar corona is close to steady state in the solar rotation frame of reference on time scales less than half a solar rotation. This assumption is closest to reality in the late declining phase of the solar cycle, which allows us to make reasonable predictions of recurrent solar wind streams even at times when the boundary conditions at $1 \mathrm{AU}$ have to be rotated from the other side of the Sun, as it will be demonstrated in this paper. At the time of opposition however our solar wind predictions do not require any assumption about the state of the solar corona, and we are able to capture the propagation of transient events like interplanetary coronal mass ejections (ICMEs) even at solar maximum, when the corona is far from steady state.

[13] In this statistical study, we use hourly solar wind data in RTN coordinates as input boundary conditions at $1 \mathrm{AU}$, obtained from the OMNI database. Higher resolution input data do not change significantly the solar wind predictions in the outer heliosphere according to our test results with 5-min and hourly ISEE 3 data. Data gaps in the input data are major sources of error in solar wind predictions. Therefore we used only the years with the highest data coverage in the OMNI solar wind data (see Table 1).

\section{Validation and Statistical Analysis \\ 3.1. Heliospheric Solar Wind Data}

[14] Ideally, one would need a uniformly distributed large statistical sample of solar wind observations between 1 and $10 \mathrm{AU}$ in the ecliptic plane to accurately determine the prediction efficiency of our solar wind propagation model as a function of different parameters such as heliocentric distance, helioecliptic longitude, or the phase of the solar cycle. Unfortunately, we can only rely on the observations of a few heliospheric missions, which are rather sparse both in space and time. In this study we used all solar wind data from the spacecraft Pioneer 10, Pioneer 11, Voyager 1, Voyager 2 and Ulysses that fulfilled all of the following conditions: the spacecrafts range was between 1 and $10 \mathrm{AU}$; the helioecliptic latitude of the spacecraft did not exceed $15^{\circ}$ of either northern or southern latitude; a full year of both solar wind plasma and magnetic field data were observed around the date of the spacecraft's apparent opposition with only minor data gaps; and the data coverage of near-Earth solar wind data was $63 \%$ or higher in the corresponding period. We restricted our validation study to solar wind observations within $10 \mathrm{AU}$ because the role of pickup ions may become important in the outermost heliosphere, and this effect is not taken into account in our physical model. Applying the above conditions results in 12 years of heliospheric data that finally proved to be sufficient to obtain some statistically significant estimates of spatial and temporal variations in the prediction efficiency, as demonstrated in the following sections. These 12 periods of solar wind data, each of them covering one full year centered to the date of the spacecraft's apparent opposition, are listed in Table 1 along with additional information including the spacecraft's ephemeredes (range and helioecliptic latitude at the beginning and at the end of the period), the corresponding near-Earth solar wind data coverage, as well as the recurrence index of near-Earth solar wind speed at the time of the apparent opposition.

[15] At this point we need to explain what we mean on apparent opposition in this paper. At the time of opposition, the Sun, the Earth, and the given spacecraft are aligned (or the Earth and the spacecraft are located on the same helioecliptic longitude), and we have the best estimates of the solar wind boundary conditions at one AU close to the helioecliptic longitude of the spacecraft. However one would expect the highest correlation between the propagated and observed solar wind variables at the spacecraft some time later, when the solar wind observed at the Earth during opposition arrives at the spacecraft, and this is what we call apparent opposition. In this study, we estimated the time of apparent opposition by adding the travel time of the solar wind between the Earth and the spacecraft to the time of opposition, assuming an average speed of $500 \mathrm{~km} / \mathrm{s}$. In the case of the 12 apparent oppositions listed in Table 1, this travel time varied between 12 and $29 \mathrm{~d}$, depending on the range of the spacecraft at the time of opposition.

[16] In order to demonstrate the spatial coverage of the solar wind data used for validation purposes, we plotted the range of the spacecraft for the 12 selected years in Figure 3 . Since the majority of the spacecraft tend to move from the inner toward the outer heliosphere, the average range (dashed line) has a slightly rising trend from about 5.5 AU to $7 \mathrm{AU}$. This should be kept in mind when we interpret the results of superposed epoch analyses in the following sections. We have a more or less good coverage of ranges (heliocentric distances) from 3.6 $\mathrm{AU}$ to $9.7 \mathrm{AU}$, including the orbits of Jupiter and Saturn. The kinks in the curves of 


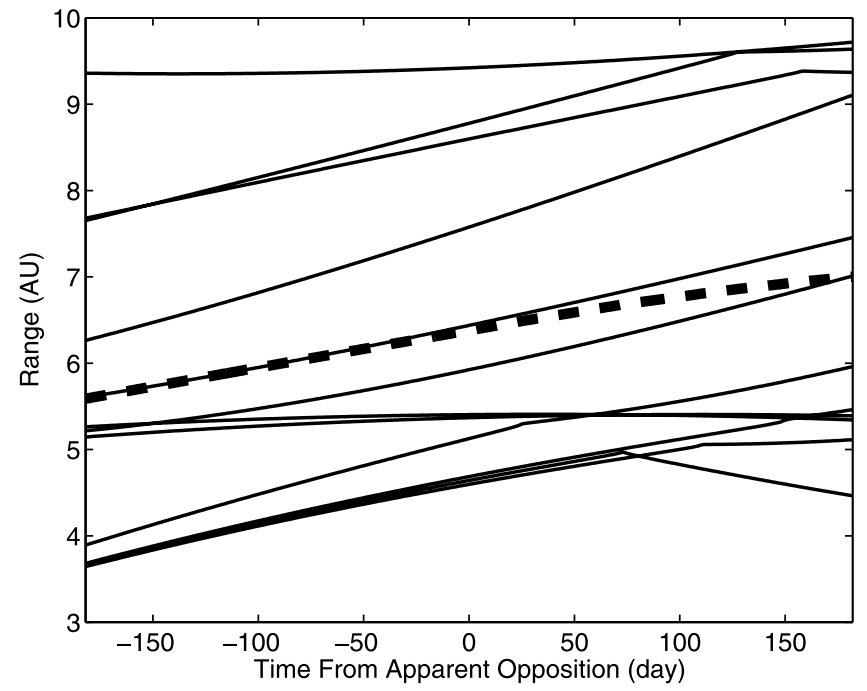

Figure 3. Ranges of heliospheric solar wind observations during the periods of validation. The average range is plotted with a dashed line.

spacecraft range indicate close encounters with either Jupiter or Saturn. The periods when a spacecraft was inside the magnetosphere of either of these planets had been excluded from the statistical analysis and were treated as data gaps.

[17] The temporal coverage of our validation data set is depicted in Figure 4. Although we use 12 years of solar wind data in our analysis, there is a substantial overlap in time between the observations of different spacecraft. For this reason, we are not able to follow the solar cycle variation of the prediction efficiency. Nevertheless, it makes sense to divide the heliospheric observations into two groups on the basis of the recurrence index of solar wind speed. The recurrence index is simply calculated as the correlation coefficient between hourly solar wind speed data in a 27-d Bartels rotation period and similar data from the previous Bartels rotation. In addition, a 13-rotation running average is applied to obtain a smoothed recurrence index. A similar recurrence index of geomagnetic activity has been introduced by Sargent [1985, 1986] and used in many studies of long-term solar wind activity [Bumba and Hejna, 1990, 1991; Hapgood, 1993; Levitin et al., 1995; Cliver et al., 1996]. Periods of high recurrence index usually occur in the late declining phase of the solar cycle often associated with recurrent high-speed solar wind streams and a tilted heliospheric current sheet. During solar activity maxima, the recurrence index is rather low because of the fast dynamic changes on the solar surface and frequent transient events like coronal mass ejections (CMEs). Thus the recurrence index of solar wind speed can be used as a measure of how steady the solar corona is on the time-scale of a solar rotation. One would naturally expect a higher prediction efficiency of solar wind variables during periods of high recurrence index, since we have to assume in our model that the boundary conditions at 1 AU do not change significantly in time within half a solar rotation. In this study, the heliospheric observations under conditions of high recurrence index ( 0.4 or higher) include Pioneer 10 and 11 data from 1973 through 1975 and Ulysses data from 2003 to 2004 , both periods falling in the declining phase of the solar cycle. Unfortunately, all these measurements were made within the range of 7 AU (see Table 1), closer to Jupiter's orbit on average. Observations under conditions of high recurrence index are not available further out in the heliosphere, close to Saturn's orbit, except for more recent solar
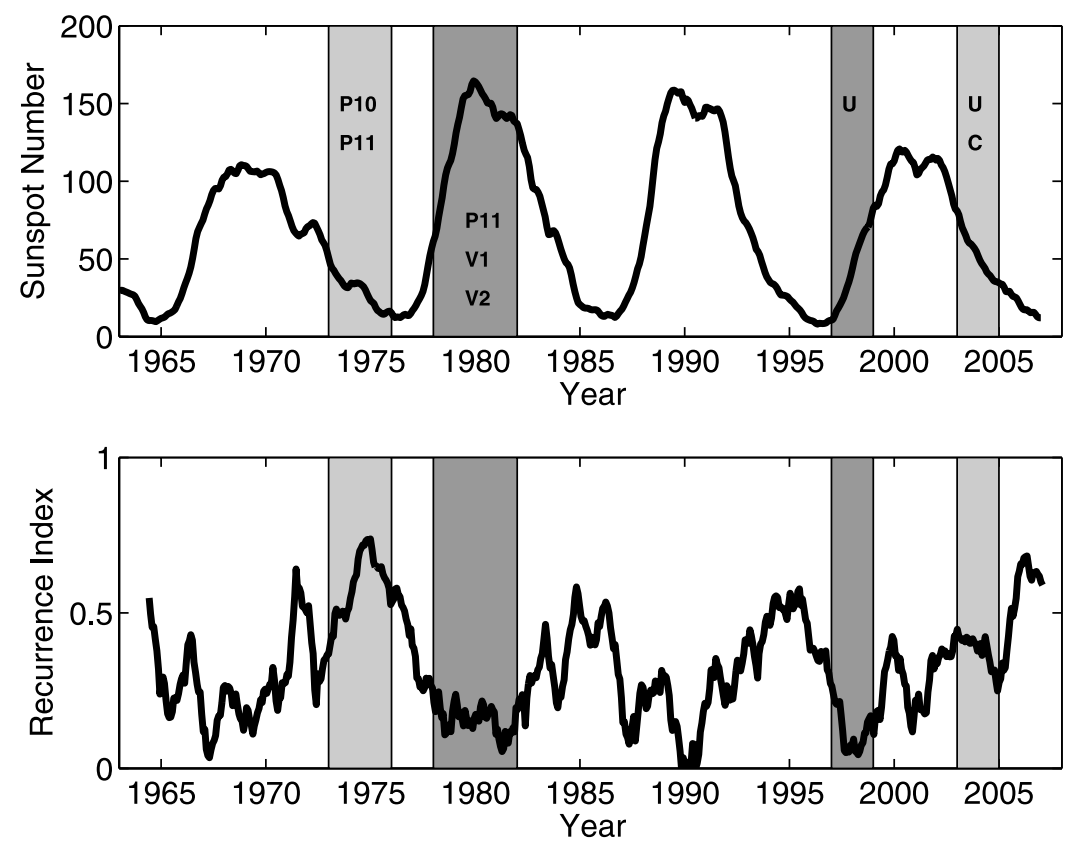

Figure 4. Monthly smoothed sunspot number (top) and the recurrence index of solar wind speed (bottom). The periods of heliospheric solar wind observations from Pioneer 10 (P10), Pioneer 11 (P11), Voyager 1 (V1), Voyager 2 (V2), Ulysses (U), and Cassini (C) under conditions of high and low recurrence index are marked with different shadings. 

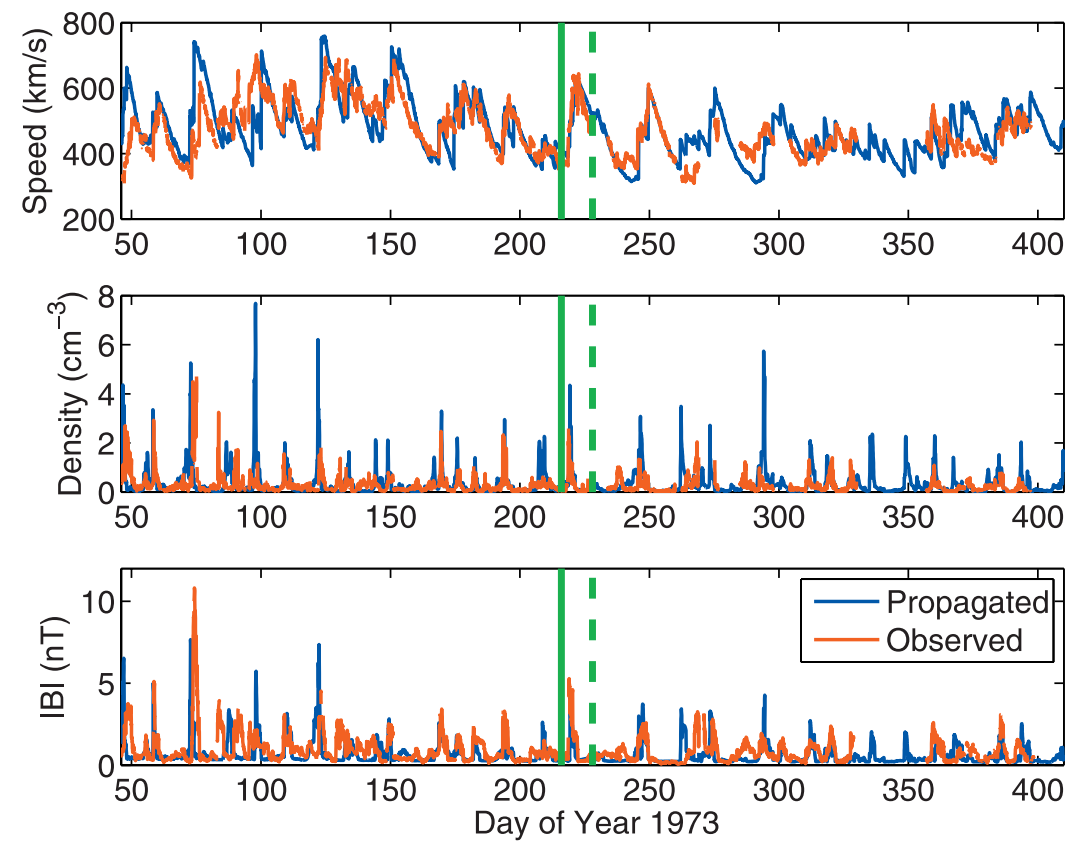

Figure 5. Propagated and observed solar wind variables at Pioneer 10 in 1973-1974 under conditions of high recurrence index.

wind data from the Cassini mission. Since we have no continuous solar wind plasma data for a full year around Cassini's apparent opposition in January 2004, we do not include the Cassini observations in all aspects of the statistical analysis. However we use 1 year of interplanetary magnetic field magnitude data from the Cassini MAG instrument in parts of the study. The heliospheric observations under conditions of low recurrence index $(0.24$ or lower) include Pioneer 11, Voyager 1, and Voyager 2 data during solar activity maximum years from 1978 through 1981, and Ulysses data from 1997 to 1998 in the early ascending phase of the solar cycle. Note that the peak recurrence index during the history of direct solar wind observations occurred in 1975, and the recurrence index of 2006 is the second highest peak, which suggests that the prediction of our solar wind propagation model must be the best during these years. On the other hand, we expect the lowest prediction efficiency during 1981, 1990, and 1998 when the recurrence index of solar wind speed reached its minima.

\subsection{Prediction Efficiency}

[18] We have performed 1-D MHD simulations for all the periods listed in Table 1, propagating the solar wind in the inertial frame from 1 AU radially outward in the heliosphere up to the range of $10 \mathrm{AU}$ along a line in the ecliptic plane, selecting a fixed helioecliptic longitude close to the spacecrafts longitude at the time of its apparent opposition. Then we extracted from the numerical solution the predicted solar wind variables (velocity, density, magnetic field, and plasma temperature) at the time-varying position of the satellite using the mapping procedure described in section 2. Here we again point out that the prediction of solar wind variables far away from the apparent opposition involves the assumption that the boundary conditions at $1 \mathrm{AU}$ do not change significantly during half a solar rotation in the frame of reference rotating with the Sun. In other words, the solar corona is close to steady state or slowly changing on the time scale of solar rotation.

[19] We compare the time series of predicted (propagated) solar wind variables with the spacecraft observed and find a generally good agreement for all variables especially in the vicinity of the spacecrafts apparent opposition. For the years with high recurrence index, the agreement is often reasonably good even half a year apart from the time of apparent opposition. On the other hand, the prediction was rather poor for some years with the lowest recurrence index. Here we show only two examples of such solar wind predictions, one for high recurrence index and the other for low recurrence index.

[20] The predicted and observed solar wind speed, density and magnetic field magnitude at Pioneer 10 in 19731974 are plotted in Figure 5. A full year of data is shown centered to the time of apparent opposition (vertical dashed line). The vertical solid line indicates the time of opposition, and the difference between the two times gives an estimate for the travel time of the solar wind from $1 \mathrm{AU}$ to the spacecraft. The fit between the predicted and observed curves is fairly impressive not only in the vicinity of the apparent opposition but also further away for all three variables. This is a typical example for a year with high recurrence index in the late declining phase of the solar cycle. Note that the coverage of the input solar wind data at the Earth was only $65 \%$ in this year. A higher solar wind data coverage would make the prediction even better.

[21] We present a typical example for a year with low recurrence index in Figure 6. This is the solar wind prediction for Voyager 1 in 1978-1979 close to solar activity maximum. Although the prediction is excellent around the time of apparent opposition (vertical dashed line), it is far from that a few months earlier or later. The data coverage of the near-Earth solar wind was as high as 

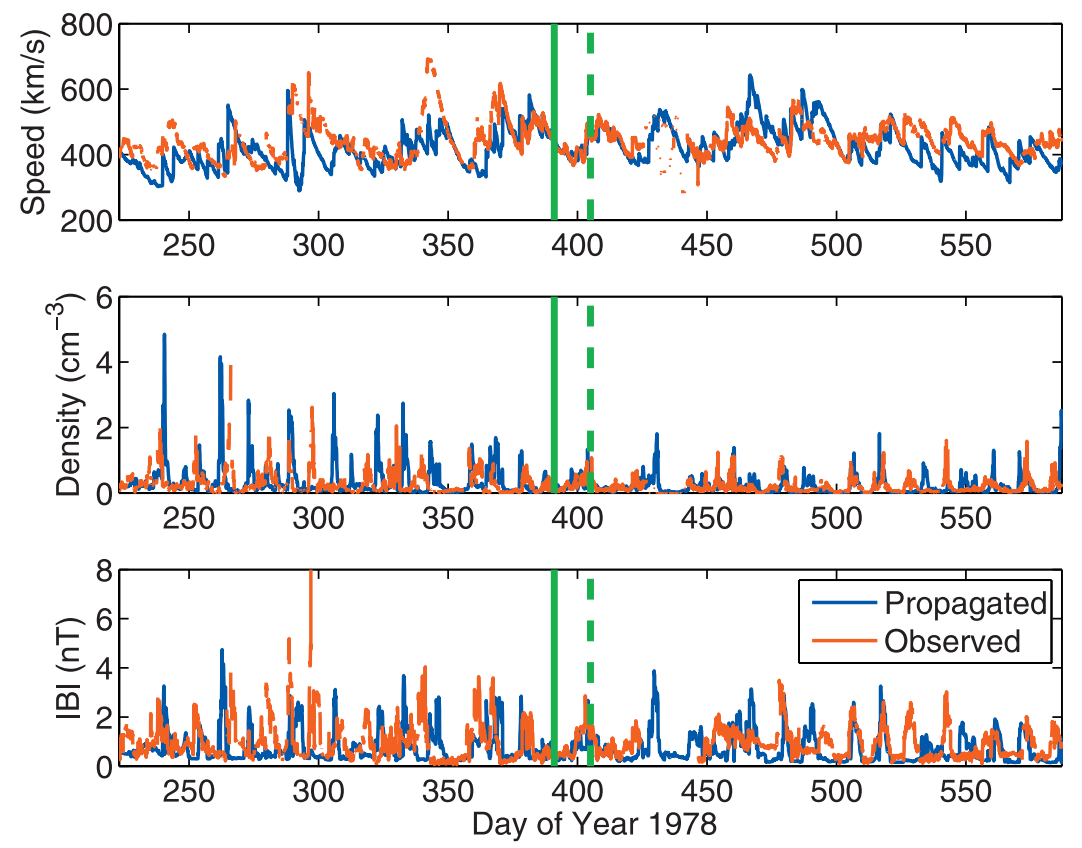

Figure 6. Propagated and observed solar wind variables at Voyager 1 in 1978-1979 under conditions of low recurrence index.

$94 \%$ in this year thus we cannot attribute the diminished correlation to an artifact of data gaps. It must have something to do with the state of the Sun and the heliosphere. During solar activity maximum a significant number of fast transient events occur in the Sun, including CMEs. With our model, we can capture such transient events only close to the opposition, which makes the prediction fairly good in the vicinity of the apparent opposition even at solar activity maximum, but at the same time it is very hard to predict solar wind boundary conditions on the opposite side of the Sun from observations near the Earth.

[22] In this section we quantify the variation of the solar wind prediction efficiency both in space and time in order to confirm our preliminary conclusions solely based on the qualitative evaluation of predicted and observed solar wind time series.

[23] To quantify the prediction efficiency as a function of time from the spacecraft's apparent opposition, we calculated correlation coefficients between predicted and observed solar wind variables in a 50-d sliding window with a time step of $5 \mathrm{~d}$ for each of the 12 periods listed in Table 1. The size of the window was chosen to cover roughly two solar rotations in the inertial frame of reference since the main part of the variance in the solar wind comes from recurrent structures. This window size is long enough to smooth out random variations in the correlation and allows sufficient time resolution at the same time. We used all the MHD variables of interest in this statistical analysis, namely the solar wind bulk flow speed, the plasma density, the magnetic field magnitude, the $B_{T}$, and $B_{N}$ components of the magnetic field in spacecraft-centered RTN coordinates as well as the plasma temperature. We did not analyze the tangential and normal velocity components and the radial magnetic field component, as the solar wind flow is mainly radially outward and the radial magnetic component cannot be propagated in 1-D MHD simulations because of the $\nabla \cdot \mathbf{B}=0$ constraint.

[24] We applied a superposed epoch analysis for the years of high recurrence index ( 0.4 or higher), the years of low recurrence index ( 0.24 or lower), and all years, respectively, averaging the respective correlation coefficients. The results are shown in the three parts of Figure 7. The time from the apparent opposition is more or less proportional to the Earth's longitudinal distance from its position at the time of apparent opposition, since the Earth moves at an almost constant speed in helioecliptic longitude much faster than any of the spacecraft in this study. This means that half a year from the apparent opposition, the Earth and the spacecraft are located in opposite sides of the Sun, and we are trying to estimate the solar wind boundary conditions at $1 \mathrm{AU}$ close to the spacecrafts longitude from observations near the Earth in the other side of the Sun, which is of course difficult.

[25] The top of Figure 7 presents the average prediction efficiency (linear correlation) of solar wind variables for high recurrence index as a function of time from the apparent opposition. The highest correlation is obtained for solar wind speed $(v)$, surpassing 0.75 at the time of apparent opposition, the second best correlation is for the magnetic field magnitude $(B)$, then comes density $(\rho)$, the azimuthal component of the magnetic field $\left(B_{T}\right)$, and the plasma temperature $(T)$ in decreasing order, and finally the least predictable variable is the northward component of the magnetic field $\left(B_{N}\right)$ with correlations close to zero. The reason why the solar wind speed is predicted best probably lies in the shape of the velocity profiles. The crosscorrelation of flat or smoothly changing profiles is less sensitive to small time shifts. On the other hand, the density profiles are more like a series of narrow peaks. If there is a small timing error in our prediction, the correlation coeffi- 

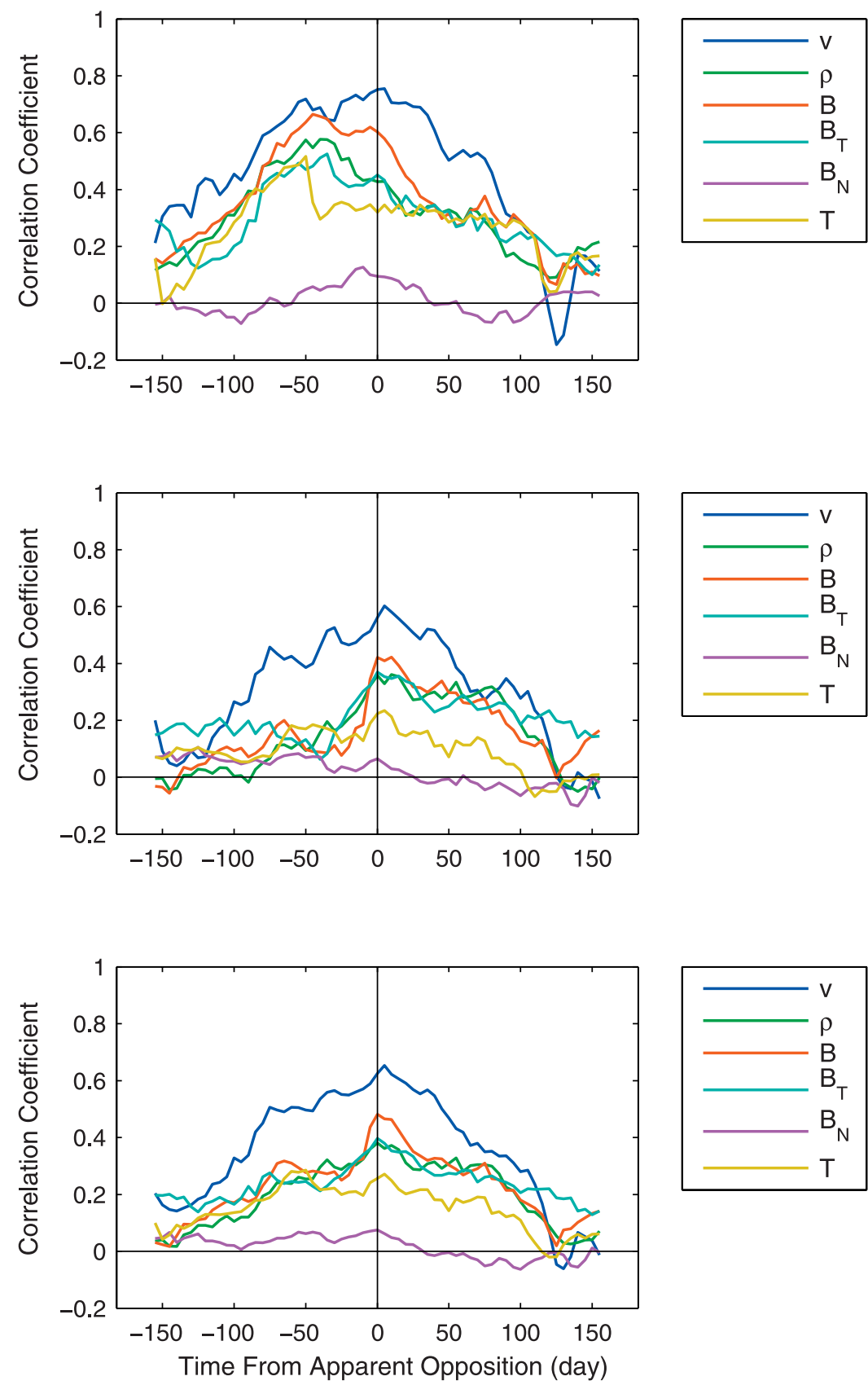

Figure 7. Average prediction efficiency (linear correlation) of solar wind variables in the function of time from apparent opposition for high recurrence index (top), for low recurrence index (middle), and for all years (bottom).

cient between predicted and observed densities can be significantly diminished. The northward IMF component is highly variable on time scales of hours or even on time scales of minutes unlike most of the other MHD variables. The cross-correlation of spiky signals is most sensitive to time shifts. Furthermore, the propagation of such fast variations to large heliocentric distances is limited by the grid resolution of our model. In addition, our model assumes solar wind propagation in the ecliptic plane and any deviation in the spacecraft's helioecliptic latitude deteriorates the predictability of the northward IMF component. These circumstances make the northward IMF component practically unpredictable.
[26] Note that the superposed epoch curves in the top of Figure 7 are based on only 4 years of data, and therefore the statistical significance of short-term variations in the correlation coefficient is rather low. Nevertheless, the correlation curves show clear decreasing trends as we move away from the time of apparent opposition, and the correlation seems to remain positive even $150 \mathrm{~d}$ from the apparent opposition, implying non-zero prediction efficiency even at this time. The only exception is $B_{N}$ that seems to be unpredictable. One can notice that some of the correlation curves tend to peak before the apparent opposition. In our opinion, this kind of bias may not be statistically significant because of the insufficient number of samples (4 years in this case). An 
opposite bias is seen in the average prediction efficiency for low recurrence index (middle of Figure 7), but the average prediction efficiency for all years with the largest number of samples (12 years) is more or less symmetric to the time of apparent opposition with clear correlation peaks just at the apparent opposition (bottom of Figure 7). It should be noted however that the average range of the spacecraft slightly increased during the superposed epoch (see Figure 3), which may introduce some real bias, provided that there is a strong dependence of prediction efficiency on the heliocentric distance. We will come back to this question later on. We can confirm that the overall prediction efficiency is significantly higher in the years with high recurrence index (top of Figure 7) than in the years with low recurrence index (middle of Figure 7), which justifies our a priori expectation. The average correlation coefficient curves calculated for all years (bottom of Figure 7) suggest a peak in the prediction efficiency just at the time of apparent opposition and relatively high correlations up to about $75 \mathrm{~d}$ before and after the time of apparent opposition. Further away, the correlations tend to drop rapidly.

[27] We also investigated the question how the prediction efficiency depends on the range (heliocentric distance) of the spacecraft. For such a study, one would need simultaneous heliospheric observations of the solar wind at different heliocentric distances close to the apparent opposition of the spacecraft. At this point we face the problem of insufficient sampling again. The best that we can do with our limited set of heliospheric observations is plot the correlation coefficients at the apparent oppositions for each spacecraft as a function of spacecraft range, trying to eliminate the temporal variation of prediction efficiency if possible. The top in Figure 8 shows the prediction efficiency of solar wind speed as a function of heliocentric distance (or spacecraft range), marking the years with high and low recurrence index with different symbols, whereas the lower panel is a similar plot for the magnetic field magnitude. The correlation coefficients were calculated in a 120-d window centered to the apparent opposition of each spacecraft, and labeled with the abbreviation of the spacecraft name in the figure. As it has been pointed out in section 3.1, the heliospheric solar wind observations under the condition of low recurrence index are biased toward smaller heliocentric distances, lacking observations around the orbit of Saturn, except for the magnetic field observations of Cassini in the years 2003 and 2004. The correlation coefficients at the apparent opposition clearly show that the prediction efficiency is significantly higher under conditions of high recurrence index than under conditions of low recurrence index at the same heliocentric distance both for the solar wind speed (top of Figure 8) and the magnetic field magnitude (bottom of Figure 8). The additional magnetic observations by Cassini suggest that this relation holds also for large heliocentric distances near the orbit of Saturn. Thus the lower overall prediction efficiency for lower recurrence index found in the superposed epoch analysis (Figure 7) cannot be attributed to the difference in the average range of the spacecraft.

[28] It is interesting to note that the correlations for low recurrence index in Figure 8 seem to increase with the heliocentric distance. The prediction efficiency at Pioneer 11 is much higher at 8-9 AU than the prediction efficiency at the Voyager spacecraft at 5-6 AU during the same time period of 1979-1980 especially in case of the magnetic field magnitude. The low correlation at Voyager 2 around 9 AU is, perhaps, an outlier from this trend, but we should keep in mind that this observation was made in 1981, just at the time of the polarity reversal of the solar main magnetic field (see Figure 4). One would expect the lowest predictability of solar wind variables in this particular phase of the solar cycle.

\subsection{The Error of Shock Arrival Times}

[29] In this section we address the accuracy of our model in predicting the arrival time of sudden changes in the solar wind dynamic pressure associated with interplanetary shocks. Discontinuities in dynamic pressure are not the only feature that might be used to measure how well the model addresses the arrival time of solar wind features however this study can be considered representative of the accuracy of the arrival of other features, magnetic sector crossings for example. We have specifically selected the solar wind dynamic pressure because of recent evidence that this solar wind property may play a significant role in determining the configuration and dynamics of the magnetosphere and auroral ionosphere of Saturn [Bunce et al., 2005, 2006; Jackman et al., 2005; Crary et al., 2005]. The prediction efficiency discussed in the previous section is based on cross-correlation coefficients between propagated and observed solar wind variables at a zero time lag, which is considered as a general measure of our model's prediction capability. However our observations of Figures 5 and 6 for each spacecraft lead us to the conclusion that often the form of the predicted solution is correct, but that the arrival time of shocks is only offset from the data. To test this idea, we calculate a cross-correlation with a tunable time lag and look for the lag at which the cross-correlation maximizes. In this section, we carry out a statistical analysis to estimate the random statistical error as well as a possible systematic error in the shock arrival times predicted with our MHD simulations.

[30] First, we calculated cross-correlation functions of hourly predicted and observed dynamic pressures $\left(\rho v^{2}\right)$ every $10 \mathrm{~d}$ for all the 12 years of solar wind data listed in Table 1 and determined the time lag of the maximum correlation in hours. We selected a 10-d window because it is long enough to reduce the random statistical fluctuations of the correlation coefficient due to the sufficiently high degree of freedom (239), and at the same time, it is short enough (shorter than half a solar rotation) to minimize the occurrence of more than one CIR or major shock in the same time window. Second, we removed the periods when the dynamic pressure changed smoothly without any clear peak or sharp gradient that could possibly be attributed to an interplanetary shock. We also removed the periods with long data gaps in the spacecraft data to eliminate artifacts as far as possible. We did not introduce any criteria for the value of the maximum correlation coefficient, which means that we included all shocks and shock-like events in the analysis, not only the best cases with the highest correlation. We restricted the calculated cross-correlation functions to a maximum time lag of $4 \mathrm{~d}$ to avoid, if possible, the correlation of shocks from two subsequent CIRs. Finally, we divided the data into two groups based on the level (either high or low) of the recurrence index of solar wind 

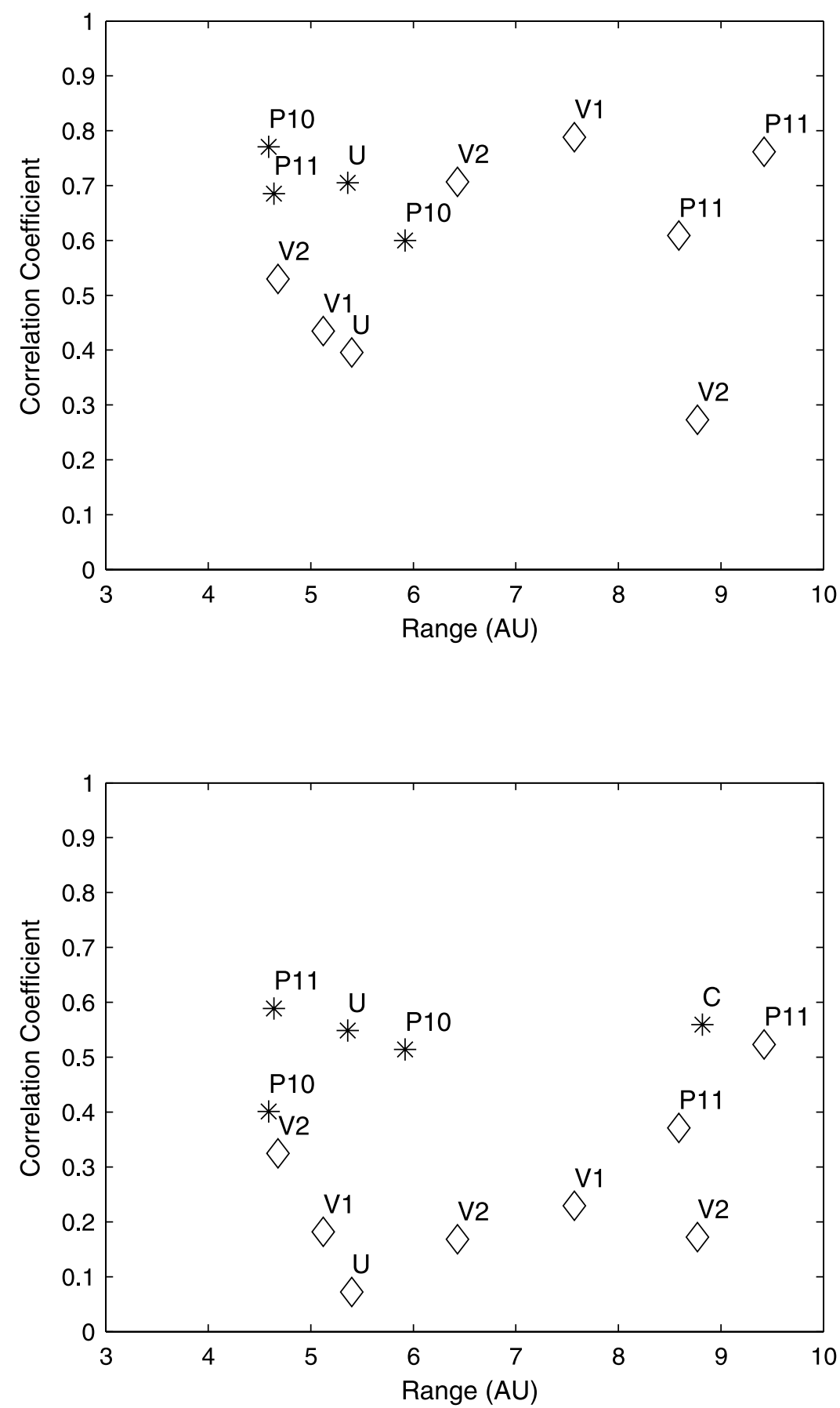

Figure 8. Prediction efficiency (linear correlation) of solar wind speed (top) and magnetic field magnitude (bottom) as a function of heliocentric distance under conditions of high (asterisk symbols) and low (diamond symbols) recurrence index, based on heliospheric solar wind data from Pioneer 10 (P10), Pioneer 11 (P11), Voyager 1 (V1), Voyager 2 (V2), Ulysses (U), and Cassini (C).

speed in the respective year, as we did in the case of the superposed epoch analysis of the prediction efficiency in section 3.2 .

[31] The time lag of the maximum correlation coefficient can be regarded as an estimate of the accuracy of the shock arrival time. A positive lag means that our model predicts the arrival of the shock at a later time than it was actually observed by the spacecraft, and correspondingly, a negative lag means that our predicted shock arrives earlier than the actual one. The predicted shock arrival times are plotted as a function of time from apparent opposition in Figure 9, separately for the years of high recurrence index (upper panel) and for the years of low recurrence index (lower panel). We would like to remind the reader that we have only 4 years of heliospheric solar wind observations under conditions of high recurrence index, whereas the solar wind observations under low recurrence index cover 8 years (see Table 1), therefore the larger number of data points in the 

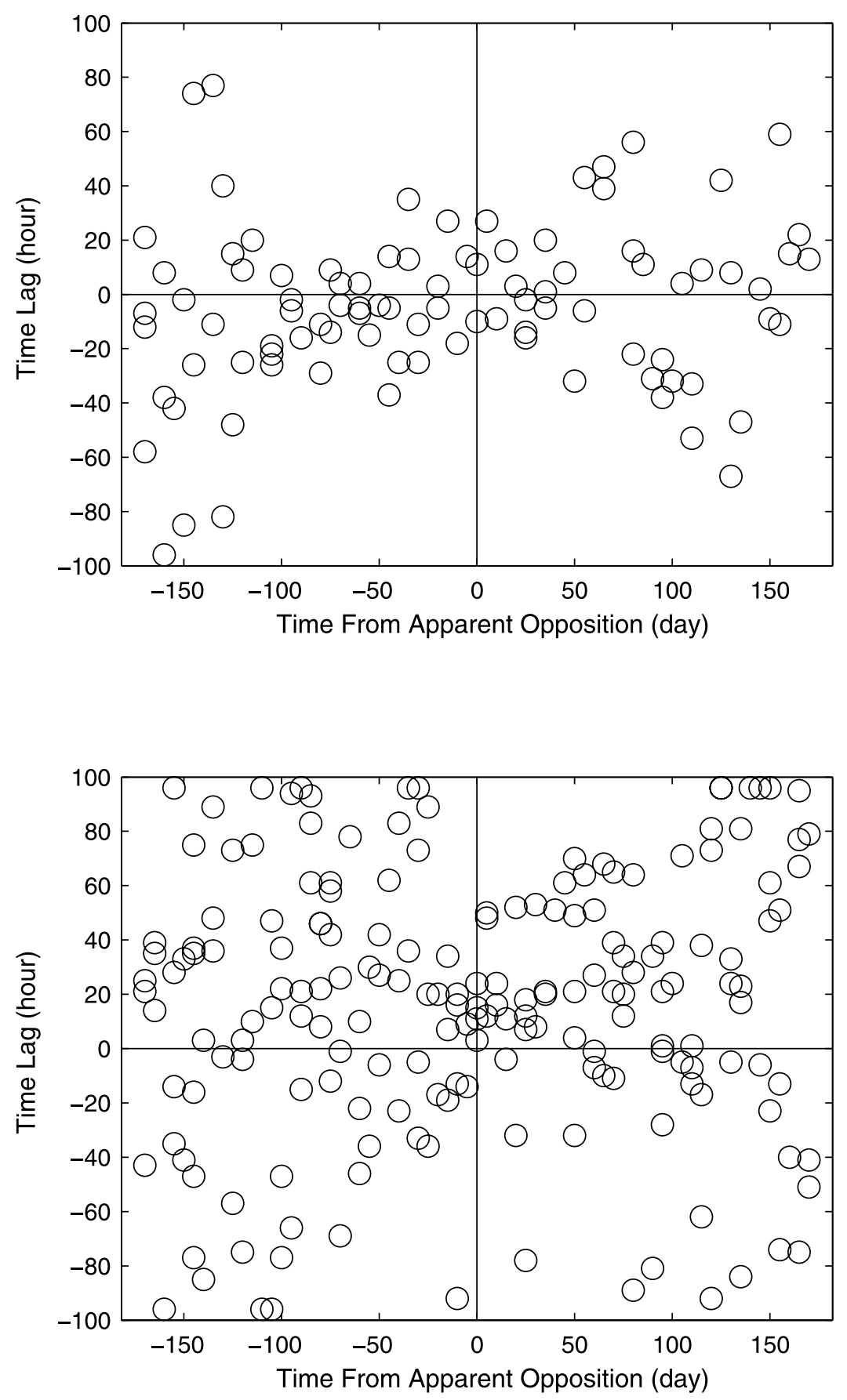

Figure 9. Shock arrival times as a function of time from apparent oppositions under conditions of high recurrence index (top) and low recurrence index (bottom). The time lags refer to the arrival times of predicted shocks with respect to the arrival times of the actual shocks observed by the spacecraft.

bottom of Figure 9 does not mean a higher occurrence rate of shocks under conditions of low recurrence index. In fact, the average number of shock arrival times per year is about the same, 23 shocks per year, for both high and low recurrence index. However we almost certainly miss a number of transient events (e.g., CMEs) in the boundary conditions at $1 \mathrm{AU}$ when the Earth is far from the Sunspacecraft line.

[32] The distribution of shock arrival times for high recurrence index (top of Figure 9) resembles a "bow tie" or "butterfly" distribution with small time lags clustering around the apparent opposition and two wings spreading more and more with increasing time from the apparent opposition. This is exactly what one would expect. Close to the apparent opposition, we have the best estimate of the boundary conditions at $1 \mathrm{AU}$, and the solar wind observations near the Earth can capture not only recurrent solar wind features but also fast transient variations. As the Earth moves away from the longitude of apparent opposition, it is getting harder and harder to estimate the boundary conditions at that fixed helioecliptic longitude, and half a year from the time of apparent opposition, when the Earth is on 

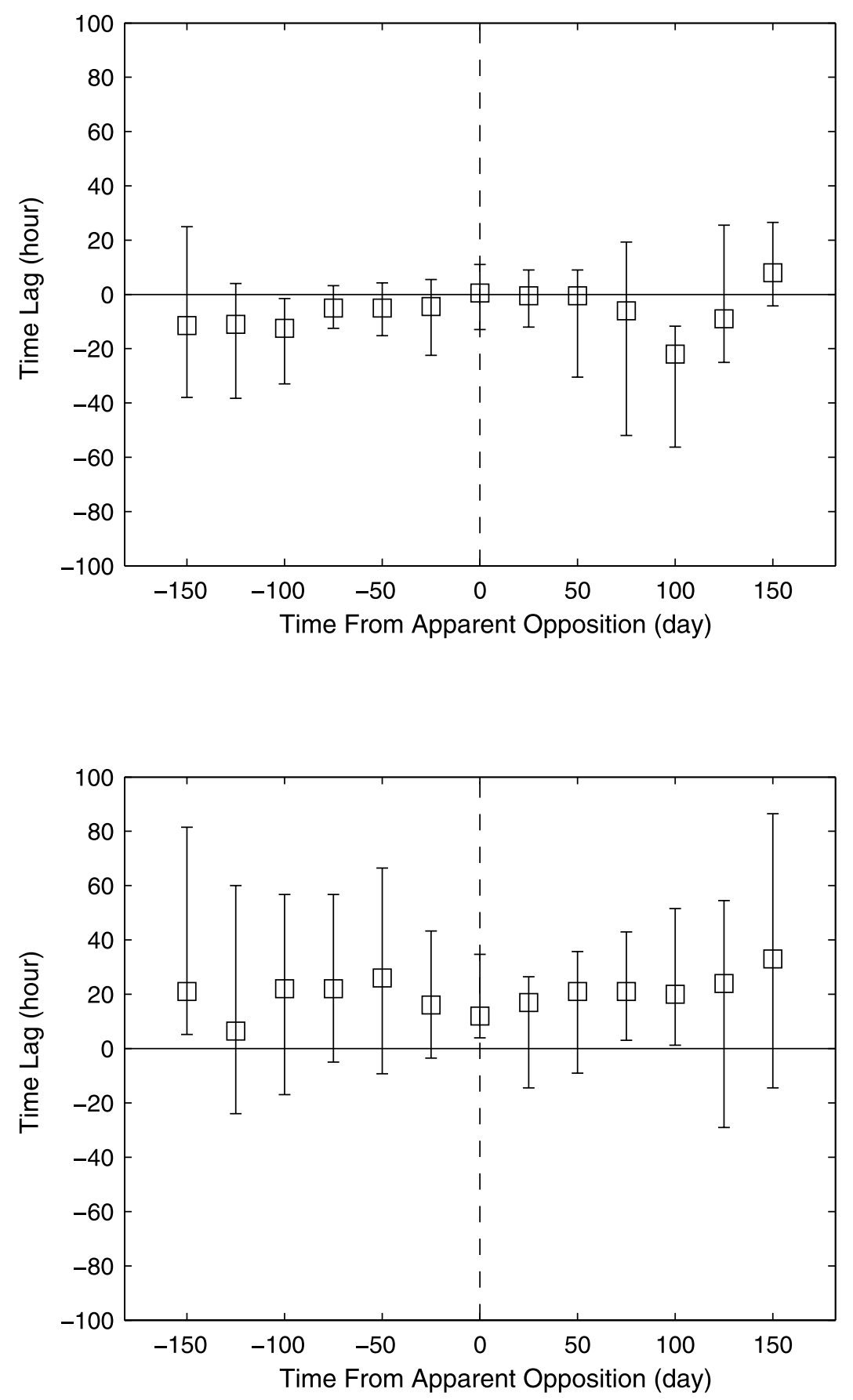

Figure 10. Estimated spread of the shock arrival time distributions shown in Figure 9. Medians and inter-quartile ranges in a 50-d sliding window are plotted with square symbols and error bars, respectively. The top/bottom refers to shock arrival times during the years with high/low recurrence index.

the other side of the Sun, we have to assume that the boundary conditions do not change significantly during half a solar rotation.

[33] The shock arrival times for low recurrence index (bottom of Figure 9) show a much larger spread including some apparent outliers. Nevertheless, the smallest time lags seem to cluster around the time of apparent opposition, as expected. We would like to warn the reader that the shock arrival times during the years of low recurrence index should be treated with extreme caution. We learned in section 3.2 that the overall prediction efficiency is significantly reduced in these years, especially further away from the time of apparent opposition. Since we did not introduce any criteria for the maximum correlations, some of the time lags may come from a rather small correlation peak in the cross-correlation function, which may not guarantee that we matched the same shock in the predicted and observed solar wind data. As mentioned already, we can easily miss some transient events in the solar wind boundary conditions during the years of low recurrence index. The missed 
transient shocks can further deteriorate the determination of shock arrival times, and can lead to outliers in the time lags.

[34] In order to quantify the spread of the shock arrival times in Figure 9, we calculated the lower and upper quartiles as well as the median of the time lags in a 50-d sliding window with a time stepping of $25 \mathrm{~d}$. The results are shown in Figure 10. The inter-quartile range in the corresponding time window is marked with error bars, whereas the median time lags are plotted with square symbols. Thus the error bars tell us, for example, that $50 \%$ of the shock arrival times are within the time lag range between -15 an 10 hours at the time of apparent opposition during years with high recurrence index (see top of Figure 10). In case of non-Gaussian distributions with outliers, it is more appropriate to describe the spread of the data with percentiles rather than with the average and the standard deviation, as the former are less sensitive to outliers. During the years of high recurrence index, the shock arrival times do not have any significant bias (top of Figure 10). The median time lags are very close to zero, especially within $75 \mathrm{~d}$ from the apparent opposition, and the inter-quartile range is gradually increasing as we move away from the apparent opposition in both directions, as one would expect. The distribution of shock arrival times during the years of low recurrence index (bottom of Figure 10), on the other hand, is apparently biased toward positive time lags. All the medians are positive, showing a slightly increasing trend as a function of time difference from the apparent opposition. This indicates a systematic error in the prediction of shock arrival times during the years of low recurrence index. Our predictions tend to be late under such conditions, or in other words, the actual shocks arrive at the spacecraft earlier than predicted, which may suggest a shock acceleration mechanism not taken into account in our MHD model. The spread of shock arrival times for low recurrence index is much larger than that for high recurrence index and the inter-quartile ranges tend to increase with the time difference from the apparent opposition, which meets our preliminary expectation.

[35] The absolute error of predicted shock arrival times can be estimated with the root mean squared deviation (RMS) from the time of arrival of the actually observed shocks. This error is plotted in Figure 11 for both high and low recurrence index. Note that the error curve for low recurrence index includes not only any random statistical error but also any systematic error in the prediction of shock arrival times, like the one pointed out in the previous paragraph. In addition, the outliers may artificially increase the RMS, especially during the years with low recurrence index. In case of high recurrence index, the error of shock arrival times is as small as 10-15 hours around the time of apparent opposition, not more than 24 hours within $75 \mathrm{~d}$ from the apparent opposition and gradually increasing further away. The error curve for high recurrence index is noisier than the similar curve for low recurrence index, because it is based on about half the number of observations than for low recurrence index. The error of shock arrival times for low recurrence index minimizes around 35 hours at the time of apparent opposition, as expected, and gradually increases further away from this time. We must remind the reader once again that this error includes a systematic error of 10 hours or more, as one can conclude from the

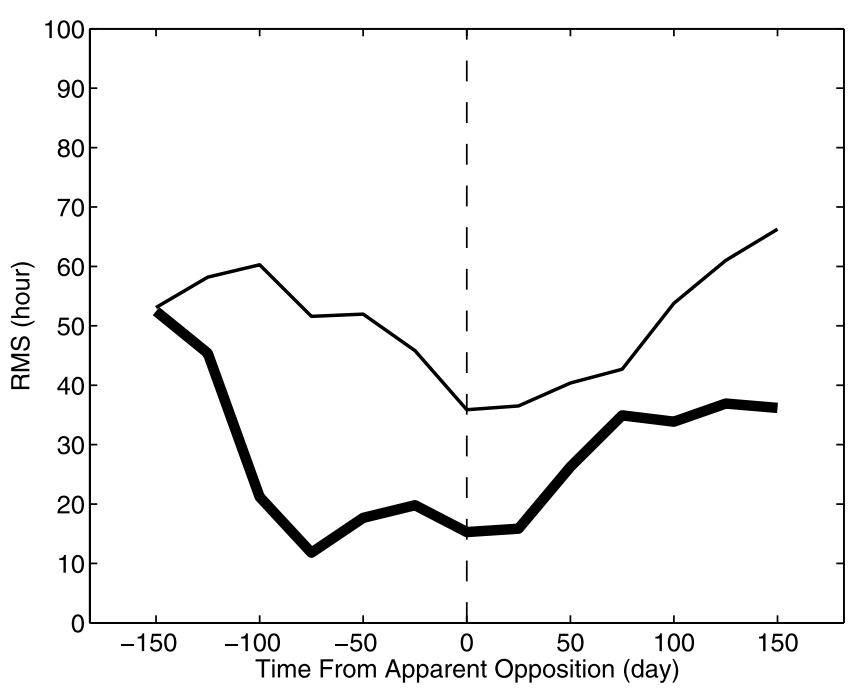

Figure 11. Estimated error of the shock arrival times plotted in the two panels of Figure 9. Root mean squared deviations (RMS) from the actually observed shock arrival times in a 50-d sliding window are plotted under the conditions of both high recurrence index (solid line) and low recurrence index (thin line).

distribution of arrival times plotted in the bottom of Figure 10.

\section{Summary and Conclusions}

[36] We have introduced a 1-D MHD model of solar wind propagation that is able to provide solar wind predictions at any location in the ecliptic plane between 1 and 10 AU. The boundary conditions at 1 AU are estimated form near-Earth solar wind observation assuming that the solar corona is in a quasi-steady state on the time scale of half a solar rotation. The time dependent MHD solution can be mapped to the location of any moving spacecraft, planet or other celestial body. The prediction capability of our model could be utilized in the planning of mission operations and different planetary observation campaigns.

[37] We have validated the solar wind propagation model with 12 years of heliospheric observations from the Voyager, Pioneer, and Ulysses spacecraft, quantifying the variations of the prediction efficiency both in space and time on a statistical basis. This is the first comprehensive validation study of its kind to the best of our knowledge. We have shown that recurrence index of solar wind speed, indicating how steady the coronal structures are on the timescale of a solar rotation, plays an important role in the temporal variation of the prediction efficiency, resulting in a solar cycle variation. Our superposed epoch analysis confirmed that under conditions of high recurrence index the efficiency of solar wind predictions is significantly higher than under conditions of low recurrence index. During solar maximum, increased solar activity makes the solar corona highly variable. ICMEs cannot be captured in the boundary conditions if they occur at a helioecliptic longitude far away from the Earth. Moreover, ICMEs significantly modify the underlying ambient solar wind, introducing significant plasma interactions also in the tangential directions, which is 
neglected in our model. In the late declining phase of the solar cycle however the solar corona at $1 \mathrm{AU}$ is close to steady state in the frame of reference rotating with the Sun. During these periods of high recurrence index, we can rotate the boundary conditions to the simulation longitude even from the other side of the Sun without introducing a large error, and consequently, our predictions will be very good throughout the year.

[38] The best predictions are expected around the apparent opposition of a spacecraft or planet, and the prediction efficiency remains reasonably good in the period between $75 \mathrm{~d}$ before and $75 \mathrm{~d}$ after apparent opposition. Significant nonzero correlation was found between observed and predicted solar wind data even at times when the Earth was separated by more than $150^{\circ}$ from the spacecraft, which means that the recurrent part of the solar wind can be predicted even if the boundary conditions are estimated from near-Earth observations at the other side of the Sun. The solar wind predictions at the apparent opposition were found to be highly reliable even at solar maximum or under conditions of low recurrence index, which means that our model is able to capture the propagation of transient events, like ICMEs as well during the periods of spacecraft alignment. The solar wind speed can be predicted to the highest accuracy (with an average linear correlation of 0.75 under conditions of high recurrence index), the second best predicted variable is the IMF magnitude, followed by the density. The plasma temperature is one of the least predictable solar wind variables, probably because of the singlefluid MHD approximation and the non-adiabatic behavior of the real solar wind.

[39] Our statistical analysis of time lags between predicted and observed shocks revealed that the error of shock arrival times is as small as $10-15$ hours within $75 \mathrm{~d}$ from apparent opposition in years with high recurrent index. This error estimate is comparable with the results of Tao et al. [2005] obtained on the basis of a much less statistical sample (12 shocks) from a single spacecraft. On the other hand, our statistical results indicate that predicted shock arrival times tend to be late by at least 10 hours during solar maximum or low recurrence index, introducing a systematic error in the shock arrival times. This may imply an additional acceleration mechanism in the solar wind during disturbed heliospheric conditions that is not taken into account in our model. A possible explanation of this extra shock acceleration is the non-radial interaction of ICMEs with CIRs, which cannot be modeled with a 1-D MHD code. This hypothesis could be tested with a 2-D version of our MHD model. Another plausible explanation is the nonadiabatic behavior of the solar wind during solar activity maximum due to additional local heating by kinetic Alfvén waves or shear driven turbulence [Matthaeus et al., 1999; Tsurutani et al., 2005; Vasquez et al., 2007]. The nonadiabatic solar wind could be modeled with a polytropic MHD model, where a polytropic equation of state is assumed in the form of $p \propto \rho^{\gamma}$ [Pizzo et al., 1995]. The polytropic index $\gamma$ can be used to mimic the volumetric heating in the corona. Empirical estimates of entropy variations in the solar wind suggest an average $\gamma$ of 1.5 [Totten et al., 1995]. Applying a polytropic index lower than $5 / 3$, our solar wind predictions during solar maximum could be further improved.
[40] Acknowledgments. This work was supported primarily through the NASA Cassini Data Analysis Program (NNX07AE54G). Work was also partially supported by the NASA Cassini Project through a Jet Propulsion Laboratory contract (JPL-1279089). BZ acknowledges support by the Hungarian Scientific Research Fund under contract NI 61013.

[41] Wolfgang Baumjohann thanks the reviewers for their assistance in evaluating this paper.

\section{References}

Bumba, V., and L. Hejna (1990), The new index of recurrence of high geomagnetic activity and its relation to the solar activity cycle, Geomagn. Aeron., 30, 669-671.

Bumba, V., and L. Hejna (1991), A new index of recurrence for long-lasting enhanced geomagnetic activity, Bull. Astron. Inst. Czech., 42, 85-90.

Bunce, E. J., S. W. H. Cowley, D. M. Wright, A. J. Coates, M. K. Dougherty, N. Krupp, W. S. Kurth, and A. M. Rymer (2005), In situ observations of a solar wind compression- induced hot plasma injection in Saturn's tail, Geophys. Res. Lett., 32, L20S04, doi:10.1029/2005GL022888.

Bunce, E. J., S. W. H. Cowley, C. M. Jackman, J. T. Clarke, F. J. Crary, and M. K. Dougherty (2006), Cassini observations of the interplanetary medium upstream of Saturn and their relation to the Hubble Space Telescope aurora data, Adv. Space Res., 38, 806-814, doi:10.1016/ j.asr.2005.08.005.

Burlaga, L. F., V. Pizzo, A. Lazarus, and P. Gazis (1985), Stream dynamics between 1 AU and 2 AU-A comparison of observations and theory, J. Geophys. Res., 90, 7377-7388.

Cliver, E. W., V. Boriakoff, and K. H. Bounar (1996), The 22-year cycle of geomagnetic and solar wind activity, J. Geophys. Res., 101, 27,09127,110 .

Crary, F. J., et al. (2005), Solar wind dynamic pressure and electric field as the main factors controlling Saturn's aurorae, Nature, 433, 720-722, doi: $10.1038 /$ nature 03333 .

Dryer, M., Z. K. Smith, E. J. Smith, J. D. Mihalov, J. H. Wolfe, R. S. Steinolfson, and S. T. Wu (1978a), Dynamic MHD modeling of solar wind corotating stream interaction regions observed by Pioneer 10 and 11, J. Geophys. Res., 83, 4347-4352.

Dryer, M., Z. K. Smith, R. S. Steinolfson, E. J. Smith, J. H. Wolfe, J. D. Mihalov, P. Rosenau, and C. Candelaria (1978b), Dynamic MHD modeling of the solar wind disturbances during the August 1972 events, J. Geophys. Res., 83, 532-540.

Du, D., C. Wang, and Q. Hu (2007), Propagation and evolution of a magnetic cloud from ACE to Ulysses, J. Geophys. Res., 112, A09101, doi:10.1029/2007JA012482.

Gosling, J. T., A. J. Hundhausen, and S. J. Bame (1976), Solar wind stream evolution at large heliocentric distances-Experimental demonstration and the test of a model, J. Geophys. Res., 81, 2111-2122.

Hanlon, P. G., M. K. Dougherty, R. J. Forsyth, M. J. Owens, K. C. Hansen, G. Tóth, F. J. Crary, and D. T. Young (2004a), On the evolution of the solar wind between 1 and 5 AU at the time of the Cassini Jupiter flyby: Multispacecraft observations of interplanetary coronal mass ejections including the formation of a merged interaction region, J. Geophys. Res., 109, A09S03, doi:10.1029/2003JA010112.

Hanlon, P. G., M. K. Dougherty, N. Krupp, K. C. Hansen, F. J. Crary, D. T. Young, and G. Tóth (2004b), Dual spacecraft observations of a compression event within the Jovian magnetosphere: Signatures of externally triggered supercorotation?, J. Geophys. Res., 109, A09S09, doi:10.1029/2003JA010116.

Hapgood, M. A. (1993), A double solar cycle in the 27-day recurrence of geomagnetic activity, Ann. Geophys., 11, 248-253.

Hundhausen, A. J., and J. T. Gosling (1976), Solar wind structure at large heliocentric distances-An interpretation of Pioneer 10 observations, J. Geophys. Res., 81, 1436-1440.

Jackman, C. M., N. Achilleos, E. J. Bunce, B. Cecconi, J. T. Clarke, S. W. H. Cowley, W. S. Kurth, and P. Zarka (2005), Interplanetary conditions and magnetospheric dynamics during the Cassini orbit insertion flythrough of Saturn's magnetosphere, J. Geophys. Res., 110, A10212, doi:10.1029/2005JA011054.

Keppens, R., and J. P. Goedbloed (1999a), Numerical simulations of stellar winds, Space Sci. Rev., 87, 223-226, doi:10.1023/A:1005152602359.

Keppens, R., and J. P. Goedbloed (1999b), Numerical simulations of stellar winds: Polytropic models, Astron. Astrophys., 343, 251-260.

Keppens, R., and J. P. Goedbloed (2000), Stellar winds, dead zones, and coronal mass ejections, Astrophys. J., 530, 1036-1048, doi:10.1086/ 308395.

Levitin, A. E., V. N. Obridko, T. E. Val'chuk, S. A. Golyshev, and L. A. Dremukhina (1995), Recurrence index of planetary geomagnetic activity and its connection with the solar rotation, Geomagn. Aeron., 35, 260263. 
Matthaeus, W. H., G. P. Zank, C. W. Smith, and S. Oughton (1999), Turbulence, spatial transport, and heating of the solar wind, Phys. Rev. Lett., $82,3444-3447$.

Parker, E. N. (1958), Dynamics of the interplanetary gas and magnetic fields, Astrophys. J., 128, 664-676.

Pizzo, V. J. (1991), The evolution of corotating stream fronts near the ecliptic plane in the inner solar system. II-Three-dimensional tilted-dipole fronts, J. Geophys. Res., 96, 5405-5420.

Pizzo, V. J., D. S. Intriligator, and G. L. Siscoe (1995), Radial alignment simulation of solar wind streams observed by Pioneers 10 and 11 in 1974 J. Geophys. Res., 100, 12,251-12,260.

Prangé, R., L. Pallier, K. C. Hansen, R. Howard, A. Vourlidas, R. Courtin, and C. Parkinson (2004), An interplanetary shock traced by planetary auroral storms from the Sun to Saturn, Nature, 432, 78-81, doi:10.1038/ nature 02986.

Richardson, J. D., K. I. Paularena, C. Wang, and L. F. Burlaga (2002), The life of a CME and the development of a MIR: From the Sun to $58 \mathrm{AU}$, J. Geophys. Res., 107(A4), 1041, doi:10.1029/2001JA000175.

Sargent, H. H., III (1985), Recurrent geomagnetic activity-Evidence for long-lived stability in solar wind structure, J. Geophys. Res., 90, 14251428 .

Sargent, H. H., III (1986), The 27-day recurrence index, in Solar Wind Magnetosphere Coupling, Astrophysics and Space Science Library, vol. 126, edited by Y. Kamide and J. A. Slavin, pp. 143-148.

Smith, Z. K., M. Dryer, R. W. Fillius, E. J. Smith, and J. H. Wolfe (1981), Compression of Jupiter's magnetosphere by the solar windReexamination via MHD simulation of evolving corotating interaction regions, J. Geophys. Res., 86, 6773-6780.

Tao, C., R. Kataoka, H. Fukunishi, Y. Takahashi, and T. Yokoyama (2005), Magnetic field variations in the Jovian magnetotail induced by solar wind dynamic pressure enhancements, J. Geophys. Res., 110, A11208, doi:10.1029/2004JA010959.

Tóth, G. (1996), A general code for modeling MHD flows on parallel computers: Versatile advection code, Astrophys. Lett. Commun., 34, $245-250$.
Totten, T. L., J. W. Freeman, and S. Arya (1995), An empirical determination of the polytropic index for the free-streaming solar wind using HELIOS 1 data, J. Geophys. Res., 100, 13-17.

Tsurutani, B. T., G. S. Lakhina, J. S. Pickett, F. L. Guarnieri, N. Lin, and B. E. Goldstein (2005), Nonlinear Alfvén waves, discontinuities, proton perpendicular acceleration, and magnetic holes/decreases in interplanetary space and the magnetosphere: intermediate shocks?, Nonlinear Proc. Geophys., 12, 321-336.

Vasquez, B. J., V. I. Abramenko, D. K. Haggerty, and C. W. Smith (2007), Numerous small magnetic field discontinuities of Bartels rotation 2286 and the potential role of Alfvénic turbulence, J. Geophys. Res., 112, A11102, doi:10.1029/2007JA012504.

Wang, C., J. D. Richardson, and J. T. Gosling (2000), A numerical study of the evolution of the solar wind from Ulysses to Voyager 2, J. Geophys. Res., 105, 2337-2344.

Wang, C., J. D. Richardson, and L. Burlaga (2001a), Propagation of the Bastille Day 2000 CME shock in the outer heliosphere, Solar Phys., 204, 413-423, doi:10.1023/A:1014293527951.

Wang, C., J. D. Richardson, and K. I. Paularena (2001b), Predicted Voyager observations of the Bastille Day 2000 coronal mass ejection, J. Geophys. Res., 106, 13,007-13,014.

Weber, E. J., and L. J. Davis (1967), The angular momentum of the solar wind, Astrophys. J., 148, 217-227.

Whang, Y. C., and L. F. Burlaga (1985), Evolution and interaction of interplanetary shocks, J. Geophys. Res., 90, 10,765-10,778.

Whang, Y. C., and L. F. Burlaga (1986), The coalescence of two merged interaction regions between 6.2 and 9.5 AU-September 1979 event, J. Geophys. Res., 91, 13,341-13,348.

K. C. Hansen and B. Zieger, Department of Atmospheric, Oceanic and Space Sciences, University of Michigan, 2455 Hayward St., Ann Arbor, MI 48109, USA. (kenhan@umich.edu; bzieger@umich.edu) 